\title{
1999 Subject index $\bullet$ Volume 5
}

\section{medure dicine}

$A \beta$, See Amyloid $\beta$ peptide

Ac-YVAD-cmk, caspase inhibition, neural graft survival, 97

Academic health centers, biomedical research squeeze, $472(\mathrm{~N})$

Academic Medicine Development Company, 6(N)

Acute myeloid leukemia

AMLI Runt domains, 1356(NV)

CD44-targeted differentiation therapy, 619(NV), 669

Adeno-associated virus

$\alpha \mathrm{V} \beta 5$ integrin co-receptor, 78, 467(L)

fibroblast growth factor receptor I co-receptor for, 7I, 467(L)

Adeno-associated virus vector

factor IX gene delivery, hemophilia B, 2 I (NV), 56,64

purification, 587

re-targeting of, genetic capsid modifications, 1052, 1438(Err)

sarcoglycan delivery, 439

Adenosine 5'-diphosphate, $\mathrm{P} 2 \mathrm{YI}$ receptor mediation of, 1199

Adenosine triphosphohydrolase, hemostasis and thrombosis modulation, 987(NV), 1010

Adenoviral vectors

instability during transport, clinical implications, 955(NT)

tissue-specific immune response, cardiac transplant implications, I | 43

glioma therapy, chronic inflammation induction, 1237(NV), 1256

Adenovirus EIA, Ewing fusion transcript EWS/FLI2 induction, 991 (NV), 1076

Advanced sleep phase syndrome, 983(NV), 1062

$\mathrm{Ag}^{+}$-resistant determinant, 183

Age-related macular degeneration, FasL role, subretinal angiogenesis, 292

Aging research center opens, $1338(\mathrm{~N})$

AIDS-Kaposi's sarcoma, IL-4 toxin treatment, 738(NV), 817

AIDS progression, SIV in vivo evolution role, 488(NV), 535

AIDS vaccine, See HIV-I vaccine

AIDS Vaccine Research Center, director appointed, 362(N)

Alcoholic cirrhosis, tumor necrosis factor- $\alpha$ role,

$\begin{array}{lrll}\text { No. I } & 1-123 & \text { No. } 7 & 713-849 \\ \text { No. } 2 & 125-243 & \text { No. } 8 & 85|-96| \\ \text { No. 3 } & 255-356 & \text { No. } 9 & 963-1087 \\ \text { No. 4 } & 357-464 & \text { No. 10 } & 1089-1214 \\ \text { No. } 5 & 465-590 & \text { No. II } & 1215-1328 \\ \text { No. 6 } & 59|-7| 1 & \text { No. I2 } & 1329-1457\end{array}$

(NV) News \& Views

(L) Letter to the Editor

(C) Commentary

(NT) New Technology

(Corr) Correction

(Err) Errata
VEGF isoforms potential use, 49I(NV), 495

Angiogenic growth factors, clinical applications,

$$
\text { I359(R) }
$$

Angiopoietins, 1359(R)

Angiotensin converting enzyme inhibitors, $1110(\mathrm{C})$

Animal rights, US universities offer courses, 973(N)

Antibiotic resistance, 147(NV), 358(L)

Antigen-presenting cells, See Dendritic cells

Antigen-specific immunosuppression, 'killer' dendritic cells, 930

Anti-inflammatory therapy, thymosin $\beta 4$ mechanism, 1424

Anti-tumor therapy, See Cancer therapy; Cancer vaccine

Anxiety, GABA $\mathrm{A}_{\mathrm{A}}$ receptor, mouse model, II31(NV)

$A P C$ mutation type, disease severity mechanism, 107I

APEC Technomart III conference, I338(N)

ApolipoproteinA-I/HDL metabolism, cubulin receptor, 656

Apoptosis, See also Neuronal apoptosis

activated $\mathrm{T}$ cells, tumor-associated antigen, 874(NV), 938

alloreactive $\mathrm{T}$ cells, transplant tolerance induction, I231(NV), 1298, 1303

amyotrophic lateral sclerosis, mice, 966(L)

Bax protein inhibition of, 832

caspase inhibitor effects, 97,298

environmental enrichment prevention of, 448 magnetic resonance spectroscopy tumor lipid detection implications, 1323(NT)

and NF- $\kappa B$ inhibition of, 412

p53 independent mechanism, atherosclerosis, 335

targeted peptides, angiogenesis toxicity, cancer, 1032

Apoptosis-linked gene 4 (ALG-4), FasL regulation, 542 Arthritis

bacterial DNA CpG motifs, 702

See also Rheumatoid arthritis

Ascorbic acid transporters, 620(NV)

Asialo-IFN- $\gamma$, hepatitis B inhibition, 577

Aspirin, angiogenesis inhibition mechanism, 1348(NV), 1418

Asthma, $\gamma \delta$ T cells role, II27(NV), I I 50

Atelocollagen, plasmid DNA delivery, 707(NT)

Atherosclerosis

CD40-CDI54 interactions, 1313

cholesteryl ester transfer protein role, 1383

p53 inactivation effect, 335 
Atypical measles, immunopathology, 629

Australia

bioethics guidelines consolidation, 973(N)

biomedical research budget increase, $598(\mathrm{~N})$

cloning report examined by parliament, 1098(N)

doctoral student drop-out problem, $72 \mathrm{I}(\mathrm{N})$

genome research facility lack of funding, 366(N)

primate center proposal, I33(N)

research papers high citation rates, $254(\mathrm{~N})$

tertiary education Green Paper, 859(N)

'Wills Report' research review, $9(\mathrm{~N})$

Autoimmune diseases

bisindolylmaleimide therapy, 19(NV), 42

CD8 cells epitope identification, Type I

diabetes, 992(NV), 1026

green fluorescent protein gene transfer, $T$ cells, 843(NT)

inhibition of, I (NV), 42,90, I346(NV), 1375

multiple sclerosis autoantibody identification,

153(NV), 170

$\mathrm{NIH}$ Office legislation, 972(N)

serum amyloid $\mathrm{P}$ component protective effect,

607(NV), 694, 852(L)

Autoimmune lymphoproliferative syndrome, 876(NV)

Autoimmune T cells

green fluorescent protein gene transfer, brain, 843(NT)

neuroprotective role, $28(\mathrm{NV}), 49$

Autonomic circuits, obesity role, 742(NV)

Azodicarbonamide, T-cell response inhibition, 947

B7 family, 1345(NV)

B7-HI, 1345(NV), 1365

Bacterial arthritis, DNA CpG motifs, 702

Bacterial meningitis, caspase inhibitor

$$
\text { neuroprotection, } 298
$$

The Baltimore case, I3(B)

Basal cell carcinoma, Ptch knockout mice, 1285

Bax protein, virus-induced neuronal apoptosis inhibition, 832

$\beta$-catenin trafficking, presenilin mutations, Alzheimer disease, I49(NV), 164

$\beta$-galactosidase-Tat fusion protein, I I33(NV)

$\beta$ I integrin, chemotherapy resistance role, lung cancer, 662

$\beta_{2}$ integrin, ICAM binding, neutrophil phagocytosis potentiation, 23I

$\beta$ tumor cell metastasis, NCAM reduced expression, 286

Bioethics

Australia harmonizes guidelines, 973(N)

and Chinese genetics, 247(L)

Declaration of Helsinki review, 1094(N)

WHO staff appointment, 1094(N)

Bioinformatics, NIH priority, 7I9(N)

Biosamples report, US bioethics commission, 974(N)

Bisindolylmaleimide, autoimmune disease therapy, I9(NV), 42

Bitter taste receptors, cloning, 38I(NV)

Bleeding time, $\mathrm{P} \mathrm{Y}_{1}$-deficient mice, 1199

Blobel, Günter, I230(N)
Blood, history of medicine and commerce, 258(B)

Blood pressure, prostaglandin $\mathrm{EP}_{2}$-deficient mice, 217

Body weight regulation

mahogany gene relevance, 374(NV)

neuropeptide $Y Y 2$ receptor role, 1188

Bolognesi, Dani, 362(N)

Bone formation, VEGF role, 617(NV),623

Bone marrow transplantation, osteogenesis imperfecta, 262(NV), 309, 466(L)

Bone regeneration, plasmid gene delivery, polymer matrix, 733(NV), 753

Books reviewed

The Baltimore Case, 13

Blood; an Epic History of Medicine and

Commerce, 258

Born That Way; Genes, Behavior, Personality, 259

The Brain and Emotion, 605

Cardiology: The Evolution of the Science and the Art, 1229

Chemokines and Cancer, 865

Dendritic Cells: Biology and Clinical Applications, 979

Designing Babies: The Brave New World of Reproductive Technology, 605

The Development of Human Gene Therapy, 728

The Devil's Workshop, 1343

Early Warning: Cases and Ethical Guidance for the Presymptomatic Testing of Genetic Diseases, 372

Has Feminism Changed Science?, 729

Heart Development, 260

A History of Molecular Biology, 140

The Human Eye: Structure and Function, 1229

Infections and Inequalities: The Modern Plagues, 727

Judging Science: Scientific Knowledge and the Federal Courts, 979

Memory: From Mind to Molecules, I 343

The Molecules Within Us, 480

Of Flies, Mice and Men, 13

Opioids in Pain Control: Basic and Clinical

Aspects, 37I

Phantoms in the Brain: Probing the Mysteries of the Human Mind, 139

The Rise and Fall of Modern Medicine, 865

The River: A Journey to the Source of HIV and AIDS, 1117

The Sceptical Witness, 980

Scientific American Molecular Neurology, 479

Textbook of Gene Therapy, 37I

Tissue Engineering of Vascular Prosthetic Grafts, 1118

Transcription Factors and Human Disease, 48I

Viruses and Human Cancer, 479

What Remains To Be Discovered: Mapping the Secrets of the Universe, the Origins of Life

and the Future of the Human Race, 258

Borrelia burgdorferi, 1346(NV), 1375

BP 897, cocaine addiction therapy, 993(NV)

Brain stem cells, differentiation, 26I(NV)

Brain tumors, oncolytic virus therapy and cyclophos phamide, rats, 881
Breast cancer, hematopoietic stem cell transplantation, 1243(NV)

Bristol Myers Squibb

African AIDS initiative, 599(N)

Distinguished Achievement Awards, 9

Brundtland, Gro Harlem, 249(N)

Busquin, Philippe, 1096(N)

C

C5a blockade, sepsis treatment, 788

Calcineurin, and heart failure, 246(L)

Calcium channel blockers, rod photoreceptor rescue, retinitis pigmentosa, 1183

Calcium

dilated cardiomyopathy role, I353(NV)

hypertrophic cardiomyopathy role, mutant $\alpha$ tropomyosins effect, 1413

and $m t D N A$ oxidative phosphorylation deficiency, 951

Calpain 3 deficiency, limb-girdle muscular dystrophy type 2A, 439, 503, 849(Err)

Canada

clinical trial approval time shortened, ethics, 1336(N)

research funds increase, $25 \mathrm{I}(\mathrm{N})$

spending spree to end 'brain drain', I336(N)

xenotransplantation research activity, 36I(N)

Cancer Research Campaign, tobacco money stand, I25(E), 129(N)

Cancer therapy

dendritic cell-NK cell cross-talk, 405

FasL counterattack, 267(NV)

NF- $\kappa B$ inhibition, 412

NSAIDs angiogenesis inhibition, I348(NV), 1418

oncolytic viruses and cyclophosphamide, 88।

pro-apoptotic peptides, angiogenesis targeting, 1032

Ras downregulation, 989 (NV)

RCASI tumor-associated antigen role, 938

telomerase reverse transcriptase target,

II29(NV), I I64

tumor necrosis factor- $\alpha$ neutralization, 828

Cancer vaccine

antigen-presenting cell modulation, 780

CD40-stimulation component, 774

clinical trials design strategies, I I24(NV), I I7 I

naked, self-replicating RNA, 823

patient specificity plus granulocyte-monocyte colony

stimulating factor, I I 24(NV), I I7 I

Cannabinergic pain modulatory system, 1243(NV)

Capsid modifications, adeno-associated virus re-targeting, 1052, 1438(Err)

Carcinogenesis, natural selection role, II(C)

Cardiac failure

$\alpha$-cardiac myosin heavy chain mutation, 266(NV), 327

and calcineurin, 246(L), 266(NV)

enterovirus protease $2 \mathrm{~A}$ dystrophin cleavage, 266(NV), 320

intracellular calcium role, I353(NV)

prevention of, postmyocardial infarction, II22(NV), 1135

VEGF isoforms potential use, 49I(NV), 495 
Cardiac function, nitric oxide synthase modulation of, 273(NV), 331

Cardiac imaging, functional, 237(NT)

Cardiac myocytes

$\alpha$-tropomyosin-induced calcium sensitization, 1413 nitric oxide synthase role, 273(NV),33।

Cardiac pressor agents, hemodynamics, I24I(NV)

Cardiac rupture prevention, I I22(NV), II 35

Cardiac transplantation

adenovirus-mediated gene transfer value, II43

passenger leukocytes and microchimerism function, 1292

Cardiology, 1229(B)

Cardiomyopathy

$\alpha$-cardiac myosin heavy chain mutation, 266(NV), 327

calcineurin role, 246(L), 266(NV)

enterovirus protease $2 \mathrm{~A}$ dystrophin cleavage, 266(NV), 320

Cardiovascular 'orphan' G protein receptor agonists, physiology, I24I(NV)

Cargo processing, endoplasmic reticulum, 745(R)

Caspase inhibition

bacterial meningitis neuroprotection, 298

nigral transplants increased survival, 97

Caspase-3

apoptotic cascade activation, spinal cord trauma, 943

HIV-I gene therapy, 27(NV), 29

Caspases, ALPS II genetic analysis, 876(NV)

Cathepsin $\mathrm{C}$, peridontal infection protective role, I358(N)

CCR5 co-receptor

allo-immunization downregulation of, 1004

biochemical properties, and HIV fusion, 303 , 590(Err)

HIV T-cell cytopathology, 344,592(L)

inhibitors of, HIV evolution danger,

740(NV), I09I(L)

$\mathrm{CD}^{+}$cells, See also HIV-specific CD4 ${ }^{+}$cells

azodicarbonamide inhibition of, 947

CD40 priming of, tumor vaccine enhancement, 780

cytomegalovirus US2 protein effect on, 1039

green fluorescent protein gene transfer, brain,

843(NT)

stress-induced experimental colitis role, 1178

$\mathrm{CD}^{+}$cells, See also HIV-specific $\mathrm{CD} 8^{+}$cells

antigen-specific unresponsiveness, tumors, 677

CD40 priming, anti-tumor vaccine augmentation, 774

epitope identification, type I diabetes, 992(NV), 1026

CD8 $\alpha \alpha$ receptor, cytotoxic T-cell antagonism, 399

cd39-deficient mice, hemostasis and thrombotic

reactions, $987(\mathrm{NV}), 1010$

CD40

$\mathrm{CD}^{+}$cell priming, anti-cancer vaccine augmentation,

780

CDI54 interactions, atherosclerosis progression, 1313

CTL priming, anti-cancer vaccine augmentation, 774

ymphoma eradication, CD4+ cell independence, 548

CD44-targeted differentiation therapy, acute myeloid leukemia, 619(NV), 669
CD47 ligation, chronic lymphocytic leukemia cell death induction, 1277

CD95L cDNA-transfected 'killer' dendritic cells, immunosuppression, 930

CDI54

antibodies against, allograft rejection prevention, 6I6(NV), 686

atherosclerosis progression role, 1313

Cell enrichment, 459(NT)

Cellular xenotransplantation, 852(L), 855(N)

Central nervous system

autoimmune T cells protective effect, 28(NV),47

stem cell differentiation, 26I(NV)

Cerebral ischemia, See also Stroke

NAALADase inhibition neuroprotective effect, 1396

NF- $\kappa B$ role, 439,503

Chemokines, and cancer, 865(B)

Chemoresistance

extracellular matrix protein role, lung cancer, 662

NF- $\mathrm{KB}$ role, 412

Children's Vaccine Initiative, $469(\mathrm{~N})$

Chimeric HIV-I clones, PCR-mediated recombination, 237(NT)

Chimpanzees

NIH program, I2I7(L)

Quebec sanctuary conflict, 7I8(N)

China

geneticists' ethical views, $247(\mathrm{~L})$

HIV research funding, 364(N)

HIV vaccine meeting, 1097(N)

research institutes reorganization, $857(\mathrm{~N})$

tobacco smoking epidemic, I5(NV)

US research collaboration solicited, 858(N)

Chitosan-DNA oral gene delivery, peanut allergy protection, $380(\mathrm{NV}), 387$

Cholesteryl ester transfer protein, proatherogenesis, 1383

Chondrocyte hypertrophy, VEGF expression, bone for mation, 617(NV), 623

Chromatin, serum amyloid protein control of, 607(NV), 694

Chromosome 22 sequenced, I358(N)

Chronic inflammatory disease, L-selectin mechanism, 1057

Chronic liver disease, hepatitis B virus transgenic mouse model, 907

Chronic lymphocytic leukemia, CD47 ligation effect, 1277

Circadian rhythmicity

cocaine abuse link, $98 \mathrm{I}(\mathrm{N})$

inherited tendencies, advanced sleep phase disorder,

983(NV), 1062

Cirrhosis, See Liver cirrhosis

Clinical research, 477(C), 7I4(L), 853(L)

CLOCK gene mutations, 983(NV), 1062

Cluster headache, voxel-based morphometry, 732(NV), 836

Cocaine addiction

circadian locomotor activity link, $98 \mathrm{I}(\mathrm{N})$

dopamine D3 receptor partial agonist therapy,

993(NV)

Coffee studies, Vanderbilt University institute, 252(N)
Coherent multiprobe fluorescence, 35I(NT)

Colon cancer suppression, MAP kinase inhibition effect, 736(NV), 810

Colorectal cancer

mismatch repair signaling, 1339(NV)

NSAIDs angiogenesis inhibition mechanism, I348(NV), 1418

Complement pathway inhibition, stroke damage reduc tion, 995(NV)

Conflict-of-interest problems, 7I3(E), 7I7(N)

Congenital heart defects prevention, in utero gene trans fer, lambs, 14I(NV), 176

Cooperation and competition in research, $1329(\mathrm{E})$

'Cornsweet effect', 1238(NV)

Coronary heart disease

cholesteryl ester transfer protein role, rats, 1383

UK report, $600(\mathrm{~N})$

cOX 2

intestinal immune homeostasis role, 867(NV), 900

potential anti-inflammatory properties, $62 \mathrm{I}(\mathrm{NV})$, 698

COX-2 inhibitors, 1348(NV), 1418

Coxsackievirus protease 2A, dystrophin cleavage, cardiomyopathy, 266(NV), 320

CpG motifs, bacterial arthritis DNA, 702

Creatine, amyotrophic lateral sclerosis neuroprotection, 347

Cristofalo, Vincent, I30(N)

Crozemarie, Jacques, 722

Cubilin, epithelial apoA-I/HDL metabolism role, 656

CXCR4 co-receptor

allo-immunization downregulation of, 1004

biochemical properties, and HIV fusion, 303, 590(Err)

and HIV entry inhibitors, 740(NV), 1091 (L)

HIV T-cell cytopathology, 344, 592(L)

Cyclooxygenase-2

intestinal immune homeostasis role, 867(NV), 900

potential anti-inflammatory properties, $62 \mathrm{I}(\mathrm{NV})$, 698

Cyclooxygenase inhibitors, 1348(NV), 1418

Cyclophosphamide, and oncolytic virus therapy, 88I

Cyclosporin A, tumor progression link, 382(NV), 714(N)

Cytokine therapy, neovascularization role, 434

Cytomegalovirus US2 protein, CD4+ cell evasion, 1039

Cytotoxic T cells

CD40 priming, anti-tumor vaccine augmentation, 774

FasL expression, degranulation role, 19(NV), 90

lymphoma eradication, CD40-targeted therapy, 548

SIV 'escape' hypothesis, I233(NV), 1270

soluble CD8 antagonism of, 399

D

Dahl salt-sensitive hypertensive rats, 1383

'Danger hypothesis', I232(NV), 1249

Deferiprone controversy, 2(L), 7(N), I223(N)

Demyelinating disease

autoantibody identification, MS, I53(NV), 170

and hepatitis $B$ vaccination, 964(L)

Dendritic cells

biology and clinical applications, 979(B)

endogenous activators, I232(NV), 1249 
immunosuppression induction, CD95L cDNA transfection, 930

interferon production, precursors, 868(NV), 919

NK cell anti-tumor response trigger, 405

prion protein expression, scrapie, I235(NV), 1308

transplantation tolerance-inducing strategies, 1245(R)

I-Deoxy-galactonojirimycin, I 12

Desmin-related myopathy, 25(NV)

Developing countries, tobacco smoking epidemic, I5(NV)

Dexter, Mike, 597(N)

Diabetes mellitus

miscarriage risk, 126(L)

new academic center, Novo Nordisk investment, $250(\mathrm{~N})$

orally active, insulin molecule identification, $615(\mathrm{NV})$

Diabetes mellitus, insulin-dependent

CD8 T-cell epitope identification, insulin B chain, 992(NV), 1026

$\mathrm{NAD}^{+}$depletion link, 269(NV),3।4

NOD mouse model, 60I(C)

and PARP inactivation, 269(NV),314

Diabetes Research Working Group, 364(N)

Diabetic retinopathy, 1390

Dideoxynucleoside resistance, MRP4 transporter role, 1048

Dietary antigen, intestinal immune response, COX-2 role, $867(\mathrm{NV}), 900$

DiGeorge syndrome, genetically engineered mouse model, II20(NV)

Dilated cardiomyopathy

enterovirus protease 2A dystrophin cleavage, 266(NV), 320

intracellular calcium role, I353(NV)

Diltiazem, rod photoreceptor rescue, retinitis pigmentosa, 1183

DNA enzyme, Egr-I mRNA targeting, I264, I438(Err)

DNA microarrays, See Microarray technology

DNA vaccination

dangers, 126(L)

immunodeficiency virus, 526

tuberculosis, 872(NV)

DNAVEC gene therapy, 2(L)

Dopamine D3 receptor partial agonist, cocaine addiction therapy, 993(NV)

Dopaminergic neurons

graft survival, caspase inhibition, 97

MPTP degenerative effects, iNOS role, I354(NV), 1403

Downless gene cloning, 870(NV)

Drug resistance

antibiotics, 147(NV), 358(L)

nucleoside-based antiviral drugs, 1048

Ductus arteriosus closure prevention, in utero gene transfer, I4I(NV), 176

Dystrophin cleavage, enterovirus protease 2A, cardiomyopathy, 266(NV), 320

\section{E}

E2F, vein-graft decoy role, $1353(\mathrm{~N})$

Early growth response factor-I mRNA, DNA enzyme targeting of, 1264, 1438(Err)

Eastman, Richard, 129(N), 254(Corr)

Ebola virus resistance, immunologic determinants, 373(NV), 423

Embryonic stem cells, See Human embryonic stem cells; Stem cells

Emotions, and brain, 605(B)

Endochondral ossification, vascular endothelial growth factor role, $617(\mathrm{NV}), 623$

Endoplasmic reticulum signaling integration, 745(R)

Endothelial progenitor cells, neovascularization role, 434

Enterovirus protease 2A, dystrophin cleavage, cardiomyopathy, 266(NV), 320

Env protein, amino acid variation, CTL escape, SIV, 1233(NV), 1270

Environmental enrichment, 448

Ependymal stem cells, adult brain, 26I(NV)

Epidemics, pathogen population dynamics, Streptococcus, 924

Epigallocatechin-3-gallate, 1216(L)

Epithelial cells, enrichment of, 459(NT)

Erythropoietic protoporphyria, gene therapy long-term cure, mice, 768

Estrogen, prolactin pathogenesis role, 1317

Estrogen surface receptor, I330(L)

mitogen-activated protein kinase link, I I30(NV)

Ethics, See Bioethics

Ewing tumor fusion transcript EWS/FLII, viral gene induction, 99I(NV), 1076, I33I(L)

Excitotoxic necrosis, PSI knock-in mice, neurons, 10

Experimental autoimmune encephalomyelitis, myelin damage, autoantibody identification,

53(NV), 170

Experimental colitis, stress-induced reactivation, $\mathrm{CD}^{+}$ cells role, 1178

Extracellular matrix proteins, chemotherapy resistance mechanism, 662

The eye, structure and function, I229(B)

F

Fabry disease, $\alpha$-galactosidase $A$ enhancement, 112

Factor VIII, alloantibody proteolysis, hemophilia A, 1044

Factor IX gene AAV delivery, hemophilia B, $2 \mathrm{I}(\mathrm{NV}), 56,64$

Familial adenomatous polyposis, APC somatic mutation determinant, 1071

Familial hypertrophic cardiomyopathy, See Hypertrophic cardiomyopathy

Familial platelet disorder, I356(NV)

Fas ligand

apoptosis-linked gene 4 regulation of, 542

bisindolylmaleimide apoptosis facilitation, 19(NV),42

degranulation regulation of, 19(NV),90

subretinal angiogenesis control, 292

tumor-infiltrating lymphocytes apoptosis induction,

267

Febrile seizures, persistent neuronal modification, devel oping brain, $87 \mathrm{I}(\mathrm{NV}), 888$

\section{Feeding behavior}

versus autonomic circuits, obesity, 742(NV)

neuropeptide $Y Y 2$ receptor role, 1188

Feminism, influence on science, 729(B)
Fertility, prostaglandin $\mathrm{EP}_{2}$-deficient mice, 217

Fetal gene therapy, pros and cons, 245(E), 255, 256(C)

Fetal immunization, baboons, 427

Fibrinogen-coated albumin microparticles, thrombocytopenia treatment, rabbits,

17(NV), 107

Fibroblast growth factor, prolactinoma pathogenesis, 1317

Fibroblast growth factor receptor I, adeno-associated virus 2 co-receptor, 71

Fibronectin synthesis block, in utero gene transfer, heart defect prevention, I4I(NV), 176

Financial conflicts of interest, 7I3(E), 7I7(N)

Finn2 Alzheimer disease pedigree, 1090(L)

Fludarabine, STATI signaling inhibition, 444

Food allergy protection, oral gene delivery, 380(NV), 387

Food and Drug Administration

research base strengthening, I33(N)

UK blood donation ban, 720

Forensic science, 980(B)

Freedom of Information Act, 8(N)

Friend virus vaccine, lymphocyte subset requirements, 189

Functional cardiac imaging, 237(NT)

G

$\mathrm{GABA}_{\mathrm{A}}$ receptor

and anxiety, mouse model, II 31 (NV)

febrile seizure persistent modification, developing brain, 87I(NV), 888

temporal lobe epilepsy, 590(Err)

Gallbladder tumors, transforming growth factor $\beta$ । expression, 1203

$\gamma \delta \mathrm{T}$ cells, airway responsiveness downregulation, I I27(NV), II 50

Gates, Bill, 857(N)

Gene activated matrix, and gene therapy, bone regeneration, $733(\mathrm{~N}), 753$

Gene expression, microgravity conditions, 359(L)

Gene mapping, cancer cells, 24(NV)

Gene therapy

adeno-associated virus capsid modifications, 1052 , 1438(Err)

congenital heart defect prevention in utero, 245(E), 255, 256(C)

chronic brain inflammation induction,

Adv/HSV-I-TK, I237(NV), 1256

development of, 728(B)

erythropoietic protoporphyria, mice, 768

germline risk, 245(E), 255, 256(C)

hemophilia B, AAV factor IX gene delivery, 2 I (NV), 56,64

HIV protease target, 27(NV), 29

in utero pros and cons, 245(E), 255, 256(C)

limb-girdle muscular dystrophy, 439

liver cirrhosis, hepatocyte growth factor, 226

peanut allergy protection, $380(\mathrm{NV}), 387$

textbook, 37I(B)

Gene transfer

fibronectin block, heart defect prevention, I4I(NV), 176 
green fluorescent protein, brain-specific T cells, 843(NT)

Genetic diseases, presymptomatic testing, 372(B)

Genetic testing, British insurers, 974(N)

Genome patenting, 1223

George, Charles, 597(N)

Geriatric cancer research, apathy in, 97I(N)

Germany

new rules for academic professors, 974(N)

science funding cutbacks, $97 \mathrm{I}(\mathrm{N})$

science ministry increased budget, 253(N)

Germline gene therapy, pros and cons, in utero, 245(E), $255,256(C)$

Geron Bio-Med, 595(N)

Glioma gene therapy

chronic brain inflammation complication, 1256(NV), 1256

MRS lipid detection, apoptosis detection

implications, I323(NT)

Global Alliance for Vaccines and Immunization, 1089(E)

Glucocorticoids, anti-inflammatory mediators up regulation, 1424

gP4I block, HIV entry inhibitor design, I I33(NV)

Graft-versus-host disease, 839

Grafts, See Transplantation

Granulocyte-monocyte colony-stimulating factor, lymphoma vaccination, I I 24(NV), II7I

Green fluorescent protein gene transfer, 843(NT)

Green tea, tumor invasion suppression, 1216(L)

Group A Streptococcus epidemics, pathogen dynamics, 924

H

HAART, See Highly active anti-retroviral therapy Headache, voxel-based morphometry, 732(NV), 836 Heart defects, congenital, in utero gene transfer, I4I(NV), 176

Heart development, 260(B)

Heart failure, See Cardiac failure

Heart transplantation, See Cardiac transplantation

Hematopoiesis, Runt domain role, I356(NV)

Hematopoietic stem cell transplantation, breast cancer, I243(NV)

Hemophilia A, factor VIII alloantibody proteolysis, 1044

Hemophilia B, adeno-associated virus factor IX gene delivery, $21(\mathrm{NV}), 56,64$

Hemostasis, cd39-deficient mice, 987(NV), 1010

Henney, Jane, I33(N)

Heparanase

cloning and functional characterization, 735(NV), 793, 803

pro-metastatic role, 735(NV), 793, 803

Hepatitis B

asialo-IFN- $\gamma$ inhibition of, 577

immune response, animal models, 907, II25(NV)

transgenic mouse model, liver disease, 907

Hepatitis $B$ vaccination

baboons, in utero, 426

demyelinating disease incidence, 964(L)

Hepatitis $B$ virus transgenic mouse model, chronic liver disease, 907

Hepatocyte growth factor, liver cirrhosis gene therapy, 226
HER-2/neu, androgen receptor cross-talk, prostate cancer, 264(NV), 280

Hereditary non-polyposis colorectal cancer, mismatch repair signaling, 1239(NV)

High-density lipoprotein endocytosis, cubulin receptor, 656

Highly active anti-retroviral therapy

$\mathrm{CD4}^{+}$cells latent infection, $512,518,609(\mathrm{NV})$

interleukin-2 combination therapy, 6II(NV), 65I

ongoing HIV dissemination, 1099(C)

resistance to, MRP4 transporter, 1048

T-cell production systems focus, $27(\mathrm{NV}), 83$

Hippocampal neurons

apoptosis prevention, meningitis, 298

excitotoxic necrosis, PS-I knock-in mice, 101

HIV-I CCR5 co-receptor, See CCR5 co-receptor

HIV-I chimeric clones, PCR-mediated

recombination, 237(NT)

HIV-I CXCR4 co-receptor, See CXCR4 co-receptor

HIV-I entry inhibitors

gP4 I targeting, I I33(NV)

HIV virulence increase danger, 740(NV), I09I(L), I330(L)

HIV-I infection

CTL 'escape' hypothesis, I233(NV), I 270

CXCR4/CCR5 biochemical properties link, 303

lifelong persistence, $C D 4^{+}$cells, 512

HIV-I Nef protein, See Nef protein

HIV-I pathogenesis

$\mathrm{CD}^{+}$production systems focus, $27(\mathrm{NV}), 83$

and immune response evasion, 723(C)

in vivo evolution role, 488(NV), 535

HIV-I protease, gene therapy target, 27(NV), 29

HIV-I replication

allo-immunization effect, 1004

persistence of, 723(C)

SAM68 mutants inhibition of, 635, 849(Err)

virus-specific CTLs role, 27(NV), 34

HIV-specific CD4 ${ }^{+}$cells

adoptive transfer, HIV treatment potential, 27(NV), 34

allo-immunization strategy, infectivity decrease, 1004 CCR5- and CXCR4-trophic HIV cytopathology, 344 directly measured kinetics, 27(NV), 83

HAART therapy limits, 483(NV), 512, 5I8, 609(NV)

HIV 'hideout' role, I358(N)

interleukin- I-HAART combination therapy, 6II(NV), 65I

latent infection persistence, 483(NV), 5I2, 5I8, 609(NV)

Nef protein chemotaxis and activation mediation, macrophage role, 985(NV), 997

production/destruction controversy, 27(NV), 83

HIV-specific CD8 ${ }^{+}$cells

adoptive transfer, treatment potential, 27(NV), 34

allo-immunization effect, 1004

directly measured kinetics, 27(NV), 83

HIV-I Tat protein vaccine induction of, 6I2(NV), 643

HIV-I Tat protein

blood-brain barrier permeability, I I33(NV)

vaccine use, $612(\mathrm{NV}), 643$

HIV-I therapy, See also Highly active anti-retroviral therapy cytotoxic T cell adoptive transfer, 27(NV), 34 gene therapy approach, 27(NV), 29

resistance to, MRP4 transporter, 1048

T-cell production systems focus, 27(NV), 83

HIV-I vaccine

allo-immunization strategy, 1004

Beijing meeting, 1097(N)

DNA priming and recombinant pox virus, 526

HIV replication persistence challenge, 723(C)

IAVI research initiative, $5(\mathrm{~N})$

live attenuated SIV prototype, risk, I54(NV), 194

SHIV DHI2-specific neutralizing antibodies,

I42(NV), 204, 211

South African plans, 252(N)

Tat protein use, 612(NV), 643

Holt-Oram syndrome, 853(L)

Hospital for Sick Children, deferiprone controversy, 2(L), 7(N)

HTLV-I provirus DNA, Andean mummy, 1428

Human cloning research

public mistrust, 10(N)

Roslin Institute reports, 253(N)

Human embryonic stem cells

Italian clamp-down, 720

politics, ethics, and medical progress, $1339(\mathrm{C})$

potential applications, I5I(NV), 975(C)

research ban, scientists appeal, $6(\mathrm{~N})$

therapeutic cloning, 975(C)

UK government indecision, 855(N)

US debate heats up, 366(N)

Human Frontier Science Program, 7(N)

Human genome sequencing, rivals square off, 47I(N)

Human research, ethical standards, 591 (E)

Human T-cell lymphotropic virus type I provirus DNA, Andean mummy, 1428

Huntington disease, lymphoblast apoptosis, mitochondr ial depolarization, 1194

Hyperlipidemia, cholesteryl ester transfer protein role, rats, 1383

Hypertension

cholesteryl ester transfer protein role, rats, 1383

prostaglandin $\mathrm{EP}_{2}$-deficient mice, 217

Hyperthermia-induced seizures, persistent neuronal modification, developing brain, 87I(NV),

888

Hypertrophic cardiomyopathy

$\alpha$-cardiac myosin heavy chain mutation, 266(NV), 327

$\alpha$-tropomyosin-induced calcium sensitization, 1413 calcineurin role, $246(\mathrm{~L}), 266(\mathrm{NV})$

I

ICAM- $\beta_{2}$ integrin binding, neutrophil phagocytosis potentiation, 23I

I $B \alpha / N F-\kappa B$ pathway, limb-girdle muscular dystrophy, 439, 503, 849(Err)

Immune 'interference' effect, 565

Immunoglobulin A nephropathy prevention, uteroglobin role, mice, 1018

Immunology research, US lead threatened, $47 \mathrm{I}(\mathrm{N})$

Immunosuppressive drugs, design of, 1243(NV)

In utero gene therapy, pros and cons, 245(E), 255, 256(C) 
In utero immunization, baboons, 427

India

animal activists delay primate trial, 1098(N)

appeal to expatriate neuroscientists, $1222(\mathrm{~N})$

vaccine development program, 970(N)

World Bank backs AIDS program, 1096(N)

Infertility, prostaglandin $\mathrm{EP}_{2}$-deficient mice, 217

Inflammatory response suppression, L-selectin

mechanism, 1057

Influenza A virus, 484(NV)

China as origin of, 384(NV)

vaccine development, III9(NV), II57

Ingelfinger rule, $357(\mathrm{E})$

Institute for Global Health, 860(N)

Institute for Molecular Oncology, 973(N)

Insulin, orally active, small molecule identification,

$$
\text { 6I4(NV) }
$$

Insulin B chain autoantigen identification, type I diabetes,

$$
\text { 992(NV), } 1026
$$

Insulin-dependent diabetes mellitus, See Diabetes mellitus, insulin-dependent

Insulin-like growth factor-I receptor, VEGF relationship, retinal neovascularization, 1390

Integrins, ICAM binding, neutrophil phagocytosis potentiation, 231

Interferon type I, plasmocytoid monocyte production of, 868(NV), 919

Interleukin-I receptor, mental retardation role, II33(NV)

Interleukin-4 toxin, AIDS-Kaposi's sarcoma treatment, 738(NV),817

Interleukin-10, BI-HI stimulation of, I345(NV), I 365

Intermediate filaments, small heat shock protein chaper one requirement, 25(NV)

International AIDS Vaccine Initiative, $5(\mathrm{~N})$

Intestinal immune response, COX-2 role, 867(NV), 900

Intracytoplasmic sperm injection, 377(NV), 43I, 593(L), II33(NV)

Ion channels, II05(C)

Ireland, National Cancer Institute agreement, I22 I(N)

Ischemic brain injury, NAALADase inhibition neuropro tective effect, 1396

Ischemic cardiomyopathy, VEGF isoforms potential use, 49I(NV),495

Ischemic tissue neovascularization, endothelial progeni tor cells, 434

Istituto Superiore della Sanità, 858(N),967(L)

Italian National Research Council reform, I32(N)

Italy

biotechnology industry rebuff, 363(N)

fertility research clamp-down, $720(\mathrm{~N})$

Institute for Molecular Oncology, 973(N)

private research funding challenge, $135(\mathrm{C})$

rare diseases increased focus, $472(\mathrm{~N})$

research institute decree, 858(N),967(L)

university spending monitoring groups created, I222(N)

Ito, Yoichi, 474(N)

J

Japan

biomedical research reorganization, 365(N) clinical trial numbers boost, $860(\mathrm{~N})$

cloning regulators criticized, $122 \mathrm{I}(\mathrm{N})$

experimental tissue regulation, $473(\mathrm{~N})$

first xenotransplantation conference, 1335(N)

influenza vaccinations, 592(N)

“Millennium Project," I095(N)

Science and Technology Basic Plan debate, 597(N)

obacco tragedy, 1091

Joint inflammation downregulation, L-selectin mechanism, 1057

Joint Infrastructure Fund, biomedical benefits, 596(N)

\section{$\mathbf{K}$}

Kaposi's sarcoma, IL-4 toxin treatment, 738(NV), 817

Kidney development, 22(NV)

Kidney transplants

NIAID begins clinical tolerance trials, $470(\mathrm{~N})$

rejection prevention, antibodies against CDI54, 6I6(NV), 686

'Killer' dendritic cells, immunosuppression, 930

Knock-in PSI mice, neuron excitotoxic necrosis vulnerability, 101

Knudson's 'two-hit' hypothesis, I07I

\section{$\mathbf{L}$}

Laser capture microdissection, II7(NT)

Lasker Awards, 1 105, 111 10, 1113

Latent HIV-I infection, 5 I 2

Leech neurons, dynamic computer calculations, 722(N)

Lep expression, tissue-specific autoregulation, 895

Leptin

neuropeptide $Y \mathrm{Y} 2$ receptor role, 1188

obesity role, 742(NV)

tissue-specific Lep regulation, 895

Leukemic blast cells, CD44-targeted differentiation therapy, 619(NV), 669

Limb-girdle muscular dystrophy, 439,503,849(Err)

Lincoln, Abraham, DNA access denied, 859(N)

Lipopolysaccharide resistance, Toll-like receptor mutations, 144(NV)

Live attenuated vaccines

lymphocyte subset requirements, Friend virus, 189 multiply deleted SIV risk, 154(NV), 194, 590(Err)

Liver cirrhosis, hepatocyte growth factor gene therapy, 226

Liver disease, See Chronic liver disease

Long-extension PCR, dangers in the clinic, 965(L)

Luminance perception, 1238(N)

Lyme disease, autoantigen and T-cell epitopes identification, 1346(NV), 1375

Lymphoblast apoptosis, Huntington disease, mitochondrial depolarization, 1194

Lymphoma

CD40-targeted therapy, 548

patient-specific vaccination plus GM-CSF, I I 24(NV), II7I

Lymphotoxin pathway blockade, virus-induced shock re versal, 1370

M

M2 protein, influenza vaccine basis, III9(NV), II 57

Macrophage-tropic HIV, and CXCR4/CCR5 biochemical properties, fusion, 303, 590(Err)

Magnetic resonance imaging, neuronal fiber tracking, II33(NV)

Magnetic resonance spectroscopy, tumor lipid detection, apoptosis detection implications, 1323(NT)

Mahogany cloning, obesity application, 374(NV)

Malaria

European Commission decision surprise, I333(N)

immunity development, 272(NV), 340

Medicines for Malaria Venture critics, 1334(N)

vaccine future, Europe, 969(N)

Wellcome Trust report, I334(N)

Malawi, Africa, research center, 253(N)

MAP kinase inhibition, tumor growth suppression, 736(NV), 810

Marijuana, US research policy shift, $72 \mathrm{I}(\mathrm{N})$

MART-I-specific T cells, characterization, 677

Matrix metalloproteinase-inhibitor TIMP-I, I I22(NV), 1135

MCP Hahnemann University, $130(\mathrm{~N})$

Mdm2, Arf control of, 490(NV)

Measles immunopathology, 629

Mechanical ventilation, neural control, I433(NT)

Medical journalism, 357(E)

Medical records, US ruling, research implications, 1337(N)

Medicines for Malaria Venture, 1334(N)

Melanocortin response, POMC-null mice, obesity, 984(NV), 1066

Melanoma, tumor-associated antigen-specific T cells, 677

Mental illness, and brain science, III3(C)

Mental retardation, IL-I receptor role, II33(NV)

MERRF mutation, mitochondrial calcium signaling, 95।

Mesenchymal stem cells, osteogenesis imperfecta therapy, 262(NV), 309, 466(L)

Methicillin-resistant Staphylococcus aureus, I47(NV)

Microarray technology, and laser capture microdissection, II7(NT)

Microchimerism, allograft acceptance functional relevance, 1292

Microgravity cultures, gene expression, 359(L)

Minipellet plasma DNA delivery system, 707(NT)

Minor histocompatibility antigen-specific CTLs, graft-versus-host disease, 839

Minority groups

cancer research criticism, 254(N)

neuroscience research, $600(\mathrm{~N})$

Mismatch repair signaling, tumor development, 1239(NV)

Mitochondrial depolarization, lymphoblast apoptosis, Huntington disease, 1194

Mitochondrial DNA disorders calcium signaling defect, 951 mouse model, 957

Mitogen-activated protein kinase estrogen surface receptor link, II30(NV) inhibition of, tumor suppression, 736(NV), 810

MOG-specific autoantibodies, multiple sclerosis mechanism, I53(NV), I70

Molecular biology, history, I40(B)

Molecular medicine, 477(C), 7I4(L)

Molecular mimicry, chronic Lyme disease, I346(NV), 1375 
Monocytes, HIV-I fusion, CXCR4 biochemistry, 303, 590(Err)

Morphine, in pain control, $37 \mathrm{I}(\mathrm{B})$

Mouse model, mitochondrial DNA disease, 957

MPTP Parkinson disease model, iNOS knockout mice, I354(NV), 1403

Multiple-primer-induced overlapping amplification assay, I08I(NT)

Multiple sclerosis, myelin damage, autoantibody identification, 153(NV), 170

Mycobacterium tuberculosis, antibiotic resistance, 358(L)

Myelin basic protein-specific T cells, 28(NV), 49

Myelin oligodendrocyte glycoprotein autoantibodies, MS, I53(NV), 170

Myocardial angiogenesis, VEGF isoforms potential use, 49I(NV), 495

Myocardial infarction, cardiac rupture prevention, I I22(NV), II 35

Myopia, 879(NV)

\section{N}

NAALADase inhibition, ischemic brain injury protection, 1396

$\mathrm{NAD}^{+}$depletion, type I diabetes role, 269(NV), 3 I4

Naked RNA vaccine, cancer therapy, 823

Narcolepsy, 98I(N)

NARP mutation, mitochondrial calcium signaling, 95।

National Cancer Institute, minority group research, criticism, 254(N)

National Health Service research collaboration, 473(N)

National Heart Forum report, 600(N)

National Institutes of Health (US)

bioinformatics priority, 7I9(N)

conflict-of-interest investigation, 129(N), 254(Corr)

grant system overhaul proceeds, $1219(\mathrm{~N})$

PhD plan dropped, 1098(N)

PhD program established, 719(N)

public input, benefits of, 1097(N)

social science research importance, $600(\mathrm{~N})$

transplant tolerance project, I222(N)

Natural killer cells

FasL expression, degranulation role, 19(NV), 90

dendritic cells trigger of, antitumor response, 405

Natural selection, tumorigenesis role, I I(C)

nef-deleted SIV vaccine, I54(NV), 194

Nef protein

amino acid variation, CTL escape, SIV, I233(NV), 1270

HIV-I macrophage infection, lymphocyte chemotaxis and activation, 985(NV), 997

Neovascularization, endothelial progenitor cells, 434

Nephrin gene, 22(NV)

Neural apoptosis

Bax inhibition of, 832

caspase inhibitor prevention, meningitis, 298

environmental enrichment preventive effect, 448

NMDA receptor blockade, $270(\mathrm{NV})$

Neural cell adhesion molecule, $\beta$ tumor cell metastasis role, 286

Neural grafts, apoptosis reduction, caspase inhibition, 97

Neural regeneration, 734(NV)

Neural stem cell differentiation, adult brain, 26I(NV)

Neuropeptide $Y Y 2$ receptor, body weight and food intake regulation, 1188

Neuroprotective benefits, autoimmune T cells, 28(NV), 49

Neutrophil phagocytosis potentiation, ICAM- $\beta_{2}$ integrin binding, 231

Neutrophils, L-selectin shedding, inflammatory response suppression, 1057

Nevirapine use hurdles, developing countries, 1093

New York encephalitis virus mystery, I243(NV)

NF-кB

cell death promotion, cerebral ischemia, 554

chemoresistance role, 412

limb girdle muscular dystrophy, 439, 503

Nigral transplants, apoptosis reduction, caspase inhibition, 97

Nitric oxide synthase

cardiac function modulation, 273(NV),33।

and MPTP Parkinson disease model, knockout mice, I354(NV), 1403

NMDA receptor blockade, neural apoptosis, perinatal brain, 270(NV)

No-consent trials, 250(N)

Non-obese diabetic mouse model, 60I(C)

Nonsteroidal anti-inflammatory drugs, angiogenesis inhibition mechanism, 1348(NV), 1418

Novartis Pharma, academic research funding expanded, I337(N)

Novo Nordisk, academic research investment, 250(N)

Nucleoside-based antiviral drug resistance, MRP4 role, 1048

\section{0}

\section{Obesity}

autonomic circuits versus feeding, 742(NV)

and mahogany gene cloning, 374(NV)

melanocortin response, POMC-null mice, 984(NV), 1066

Ogden, Russel, I224(N)

Olefsky, Jerrold, 1098(N)

Olivieri, N., 2(L), 7(N), I223(N)

Oncogene-specific therapeutics, Ras downregulation, 989

Oncolytic virus therapy, and cyclophosphamide, 88।

Opioids, in pain control, 37I(B)

Optic nerve injury, autoimmune $T$ cells protective effect, 28(NV), 49

Oral polio vaccine/AIDS hypothesis, 1 I I7(B)

Oral tolerance, COX-2 role, $867(\mathrm{NV}), 900$

Orally active insulin molecule, 6I4(NV)

Orexins, $98 \mathrm{I}(\mathrm{N})$

Organ transplantation, See Transplantation

'Orphan' G protein-coupled receptor agonists, cardiovascular physiology, I24I(NV)

Orthogonal polarization spectral imaging, 1209(NT)

Osteogenesis, Runt domain role, 1356(NV)

Osteogenesis imperfecta, mesenchymal stem cell therapy, 262(NV), 309, 466(L)

Ovarian carcinoma, RCASI tumor-associated antigen role, $874(\mathrm{NV}), 938$

Oxford International Biomedical Centre, 363(N)

$\mathbf{P}$

P2Y,-deficient mice, ADP-induced platelet effects, II 99
$\mathrm{P}^{161 \mathrm{NK} 4 \mathrm{a}}$ senescence gene, rheumatoid arthritis treatment, 73 I(NV), 760

p53 inactivation, atherogenesis role, 335

Papillon-Lefevre syndrome, I358(N)

Passenger leukocytes, allograft acceptance function, 1292

Pancreatic beta-cell destruction, PARP inactivation effect, 269(NV), 314

Pancreatic beta tumor cell metastasis, NCAM reduced expression, 286

Parkinson disease

MPTP model, iNOS knockout mice, 1354(NV), 1403

neural graft survival, caspase inhibition, 97

thalamocortical "dysrhythmias," I349(NV)

PARP deficiency, type I diabetes resistance, 269(NV), 314

Patched inactivation, basal cell carcinoma tumorigenesis, 1285

PCR-mediated recombination, HIV-I chimeric clones, 237(NT)

Peanut allergy protection, oral gene delivery, 380(NV), 387

Periaqueductal gray, pain modulation, I243(NV)

Peridontal infection, cathepsin $\mathrm{C}$ protective role, I358(N)

Personality, genetics, 259(B)

Pesticide testing, humans, US subcommittee indecision, 972(N)

'Phen-fen' diet drugs, conflict-of-interest lawsuit, 7I7(N)

Pituitary tumor transforming gene, prolactinoma patho genesis, 1317

Plasmacytoid monocytes, type I interferon production, 868(NV), 919

Plasmid DNA

bone regeneration therapy, 733(NV), 753

delivery system, 707(NT)

Plasminogen activator inhibitor-I, cardiac rupture prevention, II22(NV), II35

Platelet substitutes, thrombocytopenia treatment, I7(NV), 107

Platelets

cd39-deficient mice, 987(NV), 1010

P2Y,-deficient mice, 1199

Pluripotent stem cells, See Stem cells

Pneumococcal meningitis, caspase inhibitor neuroprotection, 298

POMC-null mice, melanocortin response, obesity, 984(NV), 1066

Population-based epidemiological research, 853(L)

Population growth, I215(E)

Porcine endogenous retrovirus, FDA xenotransplants report, $855(\mathrm{~N})$

Potassium channel, $1105(\mathrm{C})$

PP32 species, prostate cancer modulation, 264(NV), 275

Precursor dendritic cells, interferon production, 868(NV), 919

Presenilin I knock-in mice, neuron excitotoxic necrosis vulnerability, 101

Presenilin mutations

accelerated neurodegeneration, transgenic mice, 560

$\beta$-catenin trafficking, Alzheimer disease, 149(NV), 164

Presidential DNA, access denied, 859(N)

President's Advisory Council on HIVIAIDS, I220(N)

PREST report, I I I (N) 
Prion protein, follicular dendritic cell production, I235(NV), 1308

Prion replication, 486(NV)

Pro-apoptotic peptides, angiogenesis targeting, cancer, 1032

Progressive subcortical gliosis, tau gene mutation, 454

Prolactinoma pathogenesis, 1317

Pro-opiomelanocortin null mice, melanocortin response, obesity, 984(NV), 1066

Prostaglandin $\mathrm{EP}_{2}$-deficient mice

reduced fertility, 217

salt-sensitive hypertension, 217

Prostate cancer

HER-2/neu-androgen receptor cross-talk, 264(NV), 280

modulation by Pp32 alternate species, 264(NV), 275, 1087(Corr)

Protein chaperones, and intermediate filament structural integrity, 25(NV)

Pseudomonas aeruginosa, vaccine strategy, 378(NV), 392

Ptch knockout mice, basal cell carcinoma tumorigenesis, 1285

Public relations, 357(E)

Pulmonary hemostasis, $\gamma \delta$ T cells role, II27(NV), II 150

Radionuclide (Ta-I78) ventriculography, 237(NT)

C-raf antisense, anti-tumor activity-correction, 127(L)

Randomized controlled clinical trials database, 1224(N)

RANTES, HIV allo-immunization effect, 1004

Rare diseases, Italy's increased focus on, 472(N)

Ras downregulation, oncogene specific therapy, 989

RCASI tumor-associated antigen, 874(NV), 938

Reactive oxygen species, thalidomide teratogenicity, 489(NV), 582, 7I5(L)

Recombinant pox virus immunization, immunodeficiency virus challenges, 526

Renal transplants

NIAID begins clinical tolerance trials, 470(N)

rejection prevention, antibodies against CDI54, 616(NV), 686

Reproductive technology, 605(B)

Research assessment exercise (RAE) score, 25I(N)

Research ethics, See Bioethics

Retinal neovascularization

FasL control of, 292

IGF-I and VEGF receptors relationship, I390

Retinitis pigmentosa, diltiazem rod photoreceptor rescue, 1183

Retinoid acid pre-treatment, UV irradiation damage prevention, 376(NV), 4I8, 849(Err)

Retroviruses, live attenuated vaccine design, 189

Rev protein, Sam68 synergy, HIV-I replication, 635, 849(Err)

RGS7, TNF- $\alpha$ upregulation of, sepsis, 877 (NV), 913

Rheumatoid arthritis, $p^{161 \text { NK4a }}$ senescence gene treatment, 73 I (NV), 760

Rho-associated kinase, tumor cell invasion role, 22

RNA anti-tumor vaccine, 823

ROCK protein kinases, tumor cell invasion role, 22।

Roizman, Bernard, $9(\mathrm{~N})$

Roslin Bio-Med, 595(N)
Roslin Institute, human cloning reports, 253(N) Ross, Russell, 475(Obit)

Rotating wall vessel, 359(L)

Runt domain, I356(NV)

Salmonella, silver resistance molecular basis, 183

Salt-sensitive hypertension, $\mathrm{PGE}_{2} \mathrm{EP}_{2}$ receptor, 217

Sam68 mutants, HIV-I inhibition, 635,849(Err)

San Raffaele Institute, I35(C)

Sarcoglycan complex restoration, AAV vector, 439

SCA8 expansion, 383(NV)

Schistosome infection, acquired immunity development. 1225(C)

Schizophrenia, Novartis Pharma research funding, I337(N)

sCR IsLex hybrid molecule, stroke treatment, 995(NV)

Scrapie pathogenesis, follicular dendritic cells role, 1235(NV), 1308

Seizure prevention, environmental enrichment link, 448

L-selectin, inflammatory response downregulation mechanism, 1057

Selection mechanism, tumorigenesis, I I(C)

Senescence gene $p^{161 N k 4 a}$, rheumatoid arthritis treatment, 73 I (NV), 760

Sepsis

treatment of, C5a blockade, 788

tumor necrosis factor-induced CNS changes, 877(NV), 913

Septic arthritis, bacterial DNA CpG motifs, 702

Septicemia, Toll-like receptor role, I44(NV)

Serum amyloid P component, 607(NV), 694, 852(L)

Shanghai Institutes for Biological Sciences, 857(N)

Signal hypothesis, I230(N)

Silver resistance, Salmonella molecular basis, 183

Simian immunodeficiency virus (SIV)

CTL 'escape' hypothesis, I233(NV), I 270

HIV-I Tat protein vaccine protection, 6I2(NV), 643

in vivo evolution, AIDS progression, 488(NV), 535

Simian immunodeficiency virus vaccine

envelope-specific antibodies, HIV-I/SIV chimeric virus, I42(NV), 204, 2 I I

live attenuated form, risk, I54(NV), 194, 590(Err)

Sin nombre virus, 1370

Skin carcinogenesis, tumor necrosis factor- $\alpha$ role, 828,1087(Err)

Sleep

circadian rhythm genetics, 983(NV), 1062 tumor necrosis factor role, sepsis, $877(\mathrm{NV}), 913$

Small cell lung cancer, chemotherapy resistance mechanism, 662

Small heat shock protein chaperone mutations, $25(\mathrm{~N})$

Smallpox stocks, research focus, $474(\mathrm{~N})$

The SNP Consortium, 47I (N)

South Africa

HIV treatment decision, pregnant women, I(E)

HIV vaccine grants awarded, I220(N)

HIV vaccine initiative, $252(\mathrm{~N})$

South African Medical Research Council, 367(C) Spain

new cancer research center, 722(N)

researchers reject xeno-moratorium, $36 \mathrm{I}(\mathrm{N})$
'Spanish' flu virus, 384(NV), 484(NV), I 35I(NV)

Spinal cord injury

caspase-3 apoptotic cascade activation, 943

transplanted stem cell effects, rats, 1410

Spinal cord regeneration, 734(NV)

Spinocerebellar ataxia-8 expansion, 383(NV)

Stanford University, cross-disciplinary biomedical research gift, I338(N)

STATI inhibition, fludarabine role, 444

Steady-state cultures, gene expression, 359(L)

Stem cells, See also Human embryonic stem cells differentiation in adult brain, 26I(NV) osteogenesis imperfecta therapy, 262(NV), 309 politics, ethics, and medical progress, $1339(\mathrm{C})$ spinal cord transplantation, rats, 1410 transplantation of, breast cancer, 1243(NV)

Streptococcus epidemics, pathogen dynamics, 924

Streptozocin-induced diabetes, 269(NV), 314

Stress-induced experimental colitis reactivation, CD4 cells role, 1178

Stroke

complement cascade inflammatory response, inhibition of, 995(NV)

NAALADase inhibition neuroprotective effect, 1396 NF- $\mathrm{KB}$ role, 439,503

Stromelysin-I repression, Tet-induced transformation, 990(NV)

Substantia nigra, See Nigral transplants

Sweet taste receptors, cloning, 38I(NV)

Systemic lupus erythematosus, serum amyloid $P$ component protective effect, 607(NV), 694, 852(L)

$\mathbf{T}$

T cells, See $\mathrm{CD}^{+}$cells; $\mathrm{CD} 8^{+}$cells apoptosis of, transplantation tolerance induction, I23 I (NV), 1298, I 303

autoimmunity inhibition, BIS VIII, I9(NV), 42 epitope identification, Lyme disease, I346(NV), 1375 FasL expression regulation, 19(NV), 90 HIV tropism, CXCR4 biochemistry, 303 neuroprotective role, 'benign autoimmunity', 28(NV),

49

priming interference, altered peptide ligands, 565 proliferation of, B7-HI co-stimulation, I345(NV) 1365

Ta- 178 radionuclide ventriculography, 237(NT)

Taste receptors, cloning, $38 \mathrm{I}(\mathrm{NV})$

TAT-Casp3 protein, 27(NV), 29

Tat protein

blood-brain barrier permeability, I I33(NV)

HIV-I vaccine, 6I2(NV), 643

Tau mutation, progressive subcortical gliosis, 454

Telomerase inhibition, human cancer cell death, I I29(NV), II 64

Temporal lobe epilepsy, GABAA receptor expression, 590(Err)

Tenureship controversy, German professors, 974

Tetracycline-induced transformation, stromelysin-I repression, 990(NV)

Thailand

AIDS drug trials, $1217(\mathrm{~L})$

animal welfare regulation, $722(\mathrm{~N})$ 
Thalamocortical rhythms, neurological disorders, I349(NV)

Thalidomide, teratogenic mechanism, 489(NV), 582, 7I5(L), 853(L)

Thrombocytopenia, platelet substitutes show promise, I7(NV), 107

Thromboembolism resistance, $\mathrm{P} 2 \mathrm{Y}_{1}$-deficient mice, 1199

Thromboregulation, cd39-deficient mice, 987(NV), 1010

Thymosin $\beta 4$ sulfoxide, anti-inflammation mechanism, 1424

Tissue engineering, and gene therapy, bone regeneration, 733(NV), 753

TNF-related apoptosis-inducing ligand, tumoricidal activity, I46(NV), 157

Tobacco control

increased activity, $85 \mathrm{I}$ (E)

settlement fund spending on, States, 1096(N)

UK, EU, and US activity, I6(NV)

World Bank report, 596(N)

Tobacco money, Cancer Research Campaign stand, 125(E), 129(N)

Tobacco settlement

cancer centers interest, US, I0(N)

and State spending, 1096(N)

Tobacco smoking

growing epidemic, I5(NV)

Japan's tragedy, I09I(L)

Toll-like receptor mutations, lipopolysaccharide resistance, $144(\mathrm{NV})$

Trachoma, antibiotic treatment modeling, 492(NV), 572

TRAIL (TNF-related apoptosis-inducing ligand), tumoricidal activity, I46(NV), I57

Transforming growth factor $\beta \mathrm{I}$, angiogenesis and tumor suppression, 1203

Transplantation

embryonic nigral tissue, caspase inhibition, 97 embryonic stem cells, spinal cord injury, 1410 NIAID clinical tolerance trials begin, $470(\mathrm{~N})$

$\mathrm{NIH}$ tolerance induction project, I222(N)

passenger leukocytes and microchimerism function, 1292

renal allografts, rejection prevention, 616(NV), 686 tolerance induction, T-cell apoptosis, 1231 (NV), 1298 , 1303

tolerance mechanisms, 1245(R)

Traumatic spinal cord injury, caspase- 3 apoptotic cascade activation, 943

Trichoblastomas, ptch knockout mice, 1285

Trinucleotide expansions, and spinocerebellar ataxia-8, 383(NV)

Tuberculosis

death and resurrection, $872(\mathrm{NV})$

DNA vaccine utility, 872(NV)

and inequality, 727(B)
Tumor-associated antigen-specific T cells, 677

Tumor cell invasion

heparanase function, 735(NV), 793, 803

Rho-associated kinase role, 221

Tumor growth suppression

green tea role, $1216(\mathrm{~L})$

transforming growth factor $\beta \mathrm{I}$, gallbladder, 1203

Tumor-infiltrating lymphocytes, apoptosis induction, FasL expression, 267

Tumor maintenance and regression, Ras mutation model, 989(NV)

Tumor metastasis

heparanase function, 735(NV), 793, 803

Rho-associated kinase role, 221

Tumor necrosis factor- $\alpha$

alcohol damage role, 1243(NV)

RGS7 upregulation, sepsis, 877(NV), 913

kin carcinogenesis role, 828, 1087(Err)

Tumor necrosis factor-related apoptosis-inducing ligand, 146(NV), 157

Tumorigenesis, natural selection role, II(C)

'Two-hit' hypothesis, familial adenomatous polyposis, I07I

Type I diabetes, See Diabetes mellitus, insulindependent

Type I interferon, plasmacytoid monocyte production of, 868(NV), 919

Tyrosinase-specific T cells, 677

\section{$\mathbf{U}$}

UFD IL mutations, DiGeorge syndrome, II20(NV)

Uganda, AIDS reduction lessons, 963(E), 1217(L)

Ultraviolet irradiation, retinoic acid pre-treatment effect, 376(NV), 418, 849(Err)

University of Minnesota

AIDS drug suit settlement, I224(N)

cocaine grant money repayment, $72 \mathrm{I}(\mathrm{N})$

Urotensin-II, I24I(NV)

Uterine carcinoma, RCASI tumor-associated antigen role, $874(\mathrm{NV}), 938$

Uteroglobin, immunoglobulin a nephropathy prevention, mice, 1018

\section{V}

Vaccination

baboons, in utero, 427

childhood safety, Congressional hearings, 970(N)

Vaccine development, See also Cancer vaccine; HIV-I vaccine

global coalition, 249(N)

global initiatives, $1089(\mathrm{E})$

India, 970(N)

R\&D investment value, $469(\mathrm{~N})$

Varmus, Harold, $1219(\mathrm{~N})$
Vascular endothelial growth factor

bone formation role, 617(NV),623

clinical applications, I359(R)

retinal neovascularization regulation by IGF-I receptor, 1390

re-vascularization potential, isoforms, 49 (NV),495

Vascular prosthetic grafts, tissue engineering, II I8(B)

Vasoconstrictor agents, hemodynamics, I24I(NV)

Viral genes, Ewing tumor fusion transcript induction, 99।(NV), 1076

Viral vector purification, 587(NT)

Virus-induced shock reversal, lymphotoxin pathway blockade, 1370

Viruses, and human cancer, 479(B)

Vitamin A deficiency, UV irradiation effect, prevention, 376(NV), 4I8, 849(Err)

Vitamin C transporters, 620(NV)

VITIT peptide, I243(NV)

Voxel-based morphometry, cluster headache, 732(NV), 836 vpr-deleted SIV vaccine, I54(NV), 194

W

WASp function, II28(NV)

Wellcome Trust

African research center, 253(N)

biomedical research strategy, 861 (C)

genome-sequencing facilities potential sites, 1097(N)

malaria research report, $1334(\mathrm{~N})$

researcher salary increase, $597(\mathrm{~N})$

Wikler, Dan, 1094

William H. Gates Foundation, 857(N)

'Wills Report', 9(N)

Wiskott-Aldrich syndrome, II28(NV)

Women

doctorates increase, American universities, 1337(N)

HIV allo-immunization, 1004

World Conference on Science, 859

World Disasters Report 1999, 860(N)

World Medical Association, medical research guidelines revision, 598(N)

\section{$\mathbf{x}$}

Xenogeneic cells, 852(L), 855(N)

Xenotransplantation

Canadian research activity, $36 \mathrm{I}(\mathrm{N})$

cellular level, 852(L)

FDA findings, PERV transmission, 855(N)

Japan hosts conference, I335(N)

Spanish researchers reject moratorium, 36I(N)

US guidelines, 476(E)

WHO hosts Web discussion, 595(N)

$\mathbf{Z}$

Ziagen patent lawsuit settlement, I224(N) 